(2) Open Access Full Text Article

REVIEW

\title{
Imatinib in the treatment of chronic myeloid leukemia: current perspectives on optimal dose
}

This article was published in the following Dove Press journal:

Blood and Lymphatic Cancer: Targets and Therapy

9 September 2015

Number of times this article has been viewed

\author{
Joanna Waclaw' \\ Tomasz Sacha' \\ Tomasz Stoklosa ${ }^{2}$ \\ 'Department of Hematology, \\ Jagiellonian University Collegium \\ Medicum, Kraków, ${ }^{2}$ Department of \\ Immunology, Medical University of \\ Warsaw, Warsaw, Poland
}

Correspondence: Tomasz Stoklosa Department of Immunology, Medical University of Warsaw, Banacha IA, Building F, 02-097 Warsaw, Poland Email tomasz.stoklosa@wum.edu.pl
Abstract: Imatinib was the first tyrosine kinase inhibitor (TKI), successfully used in a clinical setting. It inhibits activity of BCR-ABL1 oncogenic tyrosine kinase which is crucial in the pathogenesis of chronic myeloid leukemia (CML). The safety and efficacy of imatinib dose $400 \mathrm{mg}$ daily was established in several clinical studies. Nevertheless, imatinib dose escalation ( $\geq 600 \mathrm{mg}$ daily) has been widely explored as an option to improve clinical outcomes. Results of the meta-analysis comparing frontline therapy with imatinib $400 \mathrm{mg}$ daily vs high dose (HD, $\geq 600 \mathrm{mg}$ daily) in patients with chronic phase CML (CML-CP) showed that the rate of complete cytogenetic response as well as major molecular response (MMR) at 12 months was significantly higher in HD imatinib group. However, HD imatinib does not improve overall survival and progression-free survival. Thus, the routine use of HD imatinib as frontline treatment for CML-CP is not recommended. In patients with CML-CP resistant to standard dose, HD imatinib does not significantly improve patient outcomes without a prior cytogenetic response. Therefore, in second-line therapy, the current CML-CP treatment guidelines do not recommend imatinib dose escalation but the use of second-or third-generation TKIs. In the therapy of TKI-naïve patients with accelerated or blastic phase of CML, HD imatinib (400 mg twice daily) is one of the recommended standards. In case of disease progression while on imatinib, second- or third-generation TKIs should be administered.

Keywords: imatinib, standard dose, dose escalation, chronic myeloid leukemia, $B C R-A B L 1$, high dose

\section{Introduction}

Imatinib has been invented by a group of scientists led by Nicholas Lydon at Ciba-Geigy laboratories (later on merged with Sandoz to form Novartis) in the early 1990s. Clinical development of imatinib, called initially STI571 (from signal transduction inhibitor), was led by Brian Druker, although John Goldman had a substantial role in this process and the first clinical trial in patients with chronic myeloid leukemia (CML) started in 1998. In CML, a constitutively active tyrosine kinase, encoded by $B C R-A B L 1$ oncogene, is responsible for malignant transformation of hematopoietic stem cells. Imatinib activity results from competitive binding to the ATP-binding site in BCR-ABL1 kinase domain and preventing autophosphorylation of BCR-ABL1. This results in abrogation of several pathways activated by $B C R-A B L 1$ and involved in the control of the cell adhesion, cytoskeleton organization, apoptosis, DNA damage response, cell cycle and responsible for uncontrolled proliferation of leukemic cells in CML. ${ }^{1}$

The clinical safety and the effective dose of imatinib of $400 \mathrm{mg}$ once daily was established in the Phase I trial initiated in June 1998. ${ }^{2}$ In the subsequent Phase II trials, 
the effectiveness of imatinib at this dose was established. That was the base for the approval decision of imatinib for therapy of CML by the United States Food and Drug Administration (US FDA) in May 2001. ${ }^{3}$ The current management of patients with CML has substantially changed over the past 15 years, with new considerations of using a second- and third-generation tyrosine kinase inhibitors (2G-TKIs and 3G-TKIs, respectively) in patients resistant or intolerant to initial dose of imatinib and even in the first-line setting. Nevertheless, the efficacy of imatinib at a higher dose of $600 \mathrm{mg}$ daily or $800 \mathrm{mg}$ daily used in first- and second-line treatment of CML in chronic phase (CML-CP), as well as in accelerated (AP) and blast crisis/blastic phase (BP) of CML has been evaluated in several clinical trials. The understanding of the place in therapy for the various doses of imatinib could play an important role in the optimization of a patient's clinical outcome.

\section{High-dose imatinib as a frontline treatment of CML-CP}

The recommended dose of imatinib for initial therapy of CML-CP is $400 \mathrm{mg}$ daily. ${ }^{4}$ Of note, a maximum tolerated dose for imatinib has never been established. The dose of $400 \mathrm{mg}$ daily was recommended based on the original Phase I study by Druker et al as a one level higher than a dose which yielded good hematologic and cytogenetic responses (CyRs) in patients with CML-CP. ${ }^{2}$ Nevertheless, there have been some preliminary reports suggesting that higher imatinib dosage, such as 600 or $800 \mathrm{mg}$ daily, could be more effective and lead to deeper and faster cytogenetic and molecular responses than the standard dose. ${ }^{5,6}$ Before the era of 2G-TKIs, the conception of high-dose (HD) imatinib as a frontline therapy of CML-CP was widely explored. There have been many clinical trials addressing this issue. Kantarjian et al reported the study of 114 patients with newly diagnosed CML-CP treated with imatinib at the dose of $400 \mathrm{mg}$ twice daily. Compared with the standard dose, HD imatinib was significantly associated with a better rate of complete cytogenetic response (CCyR) $(P=0.0005)$, major molecular response (MMR) (defined as BCR-ABL/ ABL ratio $\leq 0.05 \%),(P=0.00001)$, and complete molecular response $(\mathrm{CMR}$, undetectable $\mathrm{BCR}-\mathrm{ABL})(P=0.001)$. HD imatinib was well tolerated but resulted in more frequent myelosuppression. ${ }^{7}$ The other study, 2-Arm Phase III trial, was conducted in 227 patients with CML-CP, previously resistant or intolerant to interferon-alpha (IFN- $\alpha$ ). The study compared the standard dose of imatinib $400 \mathrm{mg}$ daily (arm A) with HD imatinib (800 mg daily) for 6 months, followed by $400 \mathrm{mg}$ daily as a maintenance (arm B). Imatinib dosage of $800 \mathrm{mg}$ daily resulted in a significantly higher rate of CCyR and MMR at 6 months but not at 12 months as compared with the dose of $400 \mathrm{mg}$ daily. In contrast to non-hematologic toxicities, grade 3/4 hematologic toxicities were significantly more common in the HD arm B. ${ }^{8}$ In TIDEL-I study, Hughes et al used a dose-optimization approach in a clinical trial encompassing 103 patients with newly diagnosed CML-CP. In this study, patients were treated with imatinib $600 \mathrm{mg}$ daily with response monitored every 3 months. Patients who failed to achieve a complete hematologic response (CHR), major cytogenetic response (MCyR), CCyR, or MMR by 3, 6, 9, and 12 months were treated with a high dose of $800 \mathrm{mg}$ imatinib once daily. Dose interruptions were indicated for serious hematologic and non-hematologic toxicity. Using this approach, the estimated cumulative incidences of CCyR by 12 months and 24 months were $88 \%$ and $90 \%$, respectively, and MMR rates were $47 \%$ and $73 \%$, respectively. ${ }^{9}$ These response rates were superior to those achieved with standard dose imatinib. ${ }^{10,11}$ In patients who maintained a daily average dose of $600 \mathrm{mg}$ of imatinib for the first 6 months, MMR rates by 12 months and 24 months were 55\% and 77\% compared with $32 \%$ and $53 \%$ in patients averaging less than $600 \mathrm{mg}$ daily ( $P=0.037$ and 0.016$)$, respectively. Recently, the results of the second part of the TIDEL study have been published. The study encompassed 210 patients with newly diagnosed CML-CP divided into two equal cohorts. The initial therapy was imatinib $600 \mathrm{mg} /$ day in all patients. The study endpoints were defined molecular targets: BCR-ABL1 $\leq 10 \%, \leq 1 \%$, and $\leq 0.1 \%$ at 3,6 , and 12 months, respectively. Based on early reports suggesting a correlation between minimum serum imatinib concentration achieved and the likelihood of achieving CCyR and/or MMR, ${ }^{12}$ the imatinib dose was escalated to $800 \mathrm{mg}$ daily in patients with trough serum concentration $<1,000 \mathrm{ng} / \mathrm{mL}$. Cohort 1 patients who failed to achieve any molecular target were escalated to imatinib $800 \mathrm{mg}$ daily, and subsequently switched to nilotinib 400 $\mathrm{mg}$ twice daily if failed to reach the same target 3 months later. Cohort 2 patients who failed to achieve any target were switched to nilotinib directly, as well as patients with imatinib intolerance. At 2 years, $55 \%$ of patients remained on imatinib and $30 \%$ on nilotinib. MMR was achieved in $64 \%$ at 12 months and $73 \%$ at 24 months. Overall survival (OS) was $96 \%$ and transformation-free survival was $95 \%$ at 3 years. TIDEL-II represents a novel, interesting approach for the first-line management of CML-CP based on HD imatinib and selective, early switch to $2 \mathrm{G}-\mathrm{TKI} .{ }^{13} \mathrm{In}$ 
2011, Gafter-Gvili et al published a systematic review and meta-analysis of randomized controlled trials comparing frontline therapy with imatinib $400 \mathrm{mg}$ daily vs higher doses ( $\geq 600 \mathrm{mg}$ daily) in patients with CML-CP. ${ }^{14}$ The search encompassed four trials (European LeukemiaNet [ELN], TOPS, SPIRIT, and CML-Study IV), randomizing 1,673 patients. Three trials included patients of all prognostic risk groups, whereas one trial (ELN) included only high-risk patients. For risk assessment, the Euro score was used in one case (TOPS) and the Sokal score in remaining three. The assigned imatinib dose in the HD arm was $800 \mathrm{mg}$ daily in three trials and $600 \mathrm{mg}$ daily in one trial. Subjects in the standard dose arm of all four trials were allocated $400 \mathrm{mg}$ of imatinib daily. The TOPS and ELN trials had notably shorter median follow-up (17 and 26 months, respectively) than the CML-Study IV and the SPIRIT study (28-43 months and 47 months, respectively). The primary outcomes of the study were CCyR and MMR rates at 12 months. In both cases, risk ratio $(\mathrm{RR})>1$ favored the HD imatinib arm. The CCyR rate was significantly higher at 12 months in the HD arm (RR 1.17, 95\% confidence interval [CI] 1.08-1.26, four trials, $\mathrm{I}^{2}=33 \%$ ), as well as MMR (RR $1.26,95 \%$ CI $1.12-1.42$, four trials, $\mathrm{I}^{2}=0 \%$ ). However, there was no difference in all-cause mortality or disease progression to AP/BP at the end of follow-up. The study evaluated also safety outcomes: grade 3/4 hematologic and nonhematologic adverse events. In that case, RR $<1$ favored HD imatinib. Adverse events requiring treatment discontinuation were more common in the HD arm (RR 1.98, 95\% CI $1.20-3.26$, three trials, $\mathrm{I}^{2}=0 \%$ ), as were grade $3 / 4$ neutropenia and thrombocytopenia (RR 1.56, 95\% CI 1.15-2.12 and RR $1.86,95 \%$ CI 1.28-2.70, respectively). ${ }^{14}$ Results of the aforementioned studies show that HD imatinib compared with standard dose significantly improves CCyR and MMR at 12 months. However, there was no significant difference in OS and progression-free survival (PFS) at the time of publication of the studies (Table 1). ${ }^{15-18}$ Thus, the evidence to support the routine use of HD imatinib as frontline treatment for CML-CP is currently insufficient.

\section{High-dose imatinib as a second-line therapy for standard dose imatinib failure in CML-CP}

Before the 2G-TKIs have become widely used in secondline therapy, imatinib dose escalation was one of very few available options in patients with CML-CP resistant to standard dose imatinib (400 mg daily). Mechanisms of imatinib resistance that could be potentially overcome with dose escalation include, but are not limited to, over-expression of $B C R-A B L 1$ and gene amplification. Several clinical studies have tried to identify whether and which imatinib-resistant CML-CP patients might benefit from such an intervention. Marin et al reported on the outcome of 36 patients with CML-CP in CHR in whom imatinib dosage was increased when they failed to achieve CCyR. Fourteen patients (39\%) improved their CyR and seven (19\%) achieved CCyR. The responses were short lasting, and six patients (43\%) lost their best response and, at latest follow-up, only nine patients $(25 \%)$ had sustained improvement with five of them (14\%) remaining in CCyR. ${ }^{19}$ Furthermore, in the study conducted by Zonder et al, patients with CML-CP who had imatinib dose increased from $400 \mathrm{mg}$ daily to $600-800 \mathrm{mg}$ daily because of progressive disease (due to clonal evolution in majority of them) or inadequate CyR, after at least 1 year of therapy did not achieve significantly better responses. ${ }^{20}$ Six patients achieved MCyR (three complete and three partial). Two others had minor cytogenetic responses (mCyRs). Two patients with clonal evolution transiently lost the additional clonal aberrations. Almost all of the responses occurred within 6 months, and were typically $3-6$ months in duration. ${ }^{20}$ However, studies that provide rationale for HD imatinib in patients resistant to conventional dose have also been published in

Table I Summary of selected randomized clinical trials evaluating high-dose imatinib in newly diagnosed patients with CML-CP

\begin{tabular}{|c|c|c|c|c|}
\hline Clinical study & Imatinib & $\begin{array}{l}\text { Complete } \\
\text { cytogenetic }\end{array}$ & $\begin{array}{l}\text { Major molecular } \\
\text { response rate }\end{array}$ & $\begin{array}{l}\text { Estimated dosage } \\
\text { PFS/OS }\end{array}$ \\
\hline European LeukemiaNet & 400 mg/day & At 12 months $58 \%$ & At 12 months $33.3 \%$ & At 3 years PFS: $86 \% / O S: 84 \%$ \\
\hline study ${ }^{15}$ & 800 mg/day & At 12 months $64 \%$ & At 12 months $39.8 \%$ & At 3 years PFS: $88 \% / O S: 91 \%$ \\
\hline \multirow[t]{2}{*}{ TOPS study ${ }^{16}$} & 400 mg/day & At 12 months $66 \%$ & At 12 months $40.1 \%$ & At 18 months PFS: $95 \% / O S: 98.7 \%$ \\
\hline & 800 mg/day & At 12 months $70 \%$ & At 12 months $46.4 \%$ & At 18 months PFS: $97 \% / O S: 98.2 \%$ \\
\hline \multirow[t]{2}{*}{ SPIRIT'17 } & 400 mg/day + IFN- $\alpha$ & At 12 months $66 \%$ & At $12 / 24$ months $57 \% / 64 \%$ & At 2 years PFS: $96.8 \% / O S:$ NA \\
\hline & $600 \mathrm{mg} /$ day & At 12 months $65 \%$ & At I $2 / 24$ months $49 \% / 53 \%$ & At 2 years PFS: $96.9 \% / O S:$ NA \\
\hline German Study & 400 mg/day + IFN- $\alpha$ & At 3 years $78.5 \%$ & At 3 years $63 \%$ & At 3 years PFS: $94 \% / 99 \% / O S: 93 / 99 \%$ (for \\
\hline \multirow[t]{2}{*}{ Group IV ${ }^{18}$} & $800 \mathrm{mg} /$ day & At 3 years $85.2 \%$ & At 3 years $79 \%$ & $<\mathrm{I} \%$ and $\geq \mathrm{I} \%$ of $\mathrm{BCR}-\mathrm{ABL}$, respectively) \\
\hline & & & & At 2 years PFS: $80.3 \% /$ at 5 years OS: $91 \%$ \\
\hline
\end{tabular}

Abbreviations: CML-CP, chronic phase chronic myeloid leukemia; IFN- $\alpha$, interferon-alpha; NA, not available; OS, overall survival; PFS, progression-free survival. 
the literature. Kantarjian et al reported on 106 patients with newly diagnosed CML-CP who had been enrolled on the International Randomized Study of Interferon and STI571 (IRIS) trial, who began treatment with imatinib at a dose of $400 \mathrm{mg}$ daily, and who subsequently underwent dose escalation to either 600 or $800 \mathrm{mg}$ daily. Reasons for dose escalation were evaluated retrospectively based on two sets of criteria: the IRIS protocol-defined criteria and the ELN recommendations. The IRIS protocol allowed dose escalation of imatinib to $600 \mathrm{mg}$ and then, 1 month later, to $800 \mathrm{mg}$ for the following reasons: 1) failure to achieve CHR by 3 months, 2) failure to achieve at least an mCyR (36\%-65\% Philadelphia chromosome [Ph]-positive metaphases) by 12 months, 3) loss of an MCyR ( $<35 \%$ Ph-positive metaphases) at any time, and 4) progression. The ELN criteria (2009 version) recommended dose escalation of imatinib in case of failure or suboptimal response. ${ }^{21}$ The primary study endpoints were event-free survival (EFS) (events were defined as the first occurrence of death from any cause, progression to AP/BP of CML, or loss of an MCyR) and OS. The median time to dose escalation was 22 months for the entire cohort. Of the patients who had dose escalation based on IRIS protocol criteria, $86 \%$ achieved or regained their hematologic response within 12 months of dose escalation, and $42 \%$ achieved or regained a CyR. Of the patients who had dose escalation according to the ELN recommendations, 67\% achieved or regained a hematologic response within 12 months of dose escalation, and 38\% achieved or regained a CyR. Among all patients who underwent dose escalation, the rates of freedom from progression to AP/BP and OS were $89 \%$ and $84 \%$ at 3 years after dose increase, respectively. Relative to treatment with the standard imatinib dose, an increased frequency of grade $3 / 4$ adverse events was observed after dose escalation to $\geq 600 \mathrm{mg}$ daily for superficial edema, headache, abdominal pain, hemorrhage, pyrexia, anemia, and thrombocytopenia. ${ }^{22}$ The other study reported on 74 patients with hematologic failure, cytogenetic resistance, or suboptimal response to conventional imatinib dose. In all, 54 patients received imatinib dose escalation from $400 \mathrm{mg}$ to $600 \mathrm{mg}$ daily and 20 patients were dose-escalated from 400 to $800 \mathrm{mg}$ daily. An MCyR was achieved in 41 patients (72\%) who escalated imatinib dose for cytogenetic failure and in six patients (46\%) with hematologic failure. A CCyR was achieved in 27 patients (37\%): 38\% of the hematologic failure patients and $42 \%$ of the cytogenetic-resistant patients. After 3 years of follow-up, all responding patients were in sustained CCyR. The estimated 2-year PFS and OS was $87 \%$ and $85 \%$, respectively. ${ }^{23}$ In the study by Jabbour et al, the long-term efficacy of imatinib dose escalation in 84 patients with CML-CP who met the ELN criteria of failure to standard-dose imatinib was assessed. In all, 21 patients with hematologic failure and 63 with cytogenetic failure had their imatinib dose escalated from $400 \mathrm{mg}$ to $800 \mathrm{mg}$ daily $(n=72)$ or from 300 to $600 \mathrm{mg}$ daily $(n=12)$. After a median follow-up of 61 months from dose escalation, $69 \%$ remained alive. CMR (absence of $B C R-A B L 1$ transcript) was achieved in $40 \%$ patients, including $52 \%$ of patients with cytogenetic failure and $5 \%$ of those with hematologic failure. The estimated 2- and 3-year EFS and OS rates were $57 \%$ and $47 \%$, and $84 \%$ and $76 \%$, respectively. Responses were long lasting; $88 \%$ of patients with MCyR sustained their response beyond 2 years. Treatment was well tolerated, with $76 \%$ of patients at 12 months continuing to receive imatinib at $100 \%$ of the intended dose. ${ }^{24}$ Taken together, the aforementioned studies show that imatinib dose escalation appears to induce sustained responses in a subset of patients with cytogenetic failure or acquired cytogenetic resistance. In second-line therapy, HD imatinib does not significantly improve patient outcomes without a prior CyR. START-R was a Phase II randomized study comparing dasatinib with HDs of imatinib after failure of the latter at the standard dose. In all, 150 imatinib-resistant CML-CP patients were randomized 2:1 to $140 \mathrm{mg}$ daily dasatinib $(\mathrm{n}=101)$ or $800 \mathrm{mg}$ daily imatinib $(n=49)$. Crossover to the alternate treatment was permitted after confirmed progression. With a median follow-up of 15 months, CHRs were observed in $93 \%$ and $82 \%$ of patients receiving dasatinib and $\mathrm{HD}$ imatinib $(P=0.034)$, respectively. Dasatinib resulted in a higher rate of MCyR (52\%) than HD imatinib (33\%) $(P=0.023)$. This included CCyR in $40 \%$ and $16 \%$ of patients $(P=0.004)$, respectively. MMR rates were also more frequent with dasatinib ( $16 \%$ vs $4 \% ; P=0.038)$. Treatment failure (hazard ratio [HR], $0.16 ; P<0.001$ ) and PFS (HR, 0.14; $P<0.001)$ both favored dasatinib. ${ }^{25} \mathrm{~A}$ 2-year follow-up report of this study showed that dasatinib demonstrated higher rates of CHR ( $93 \%$ vs $82 \% ; P=0.034)$, MCyR (53\% vs 33\%; $P=0.017)$, and CCyR (44\% vs $18 \%$; $P=0.0025)$. At 18 months, the MCyR was maintained in $90 \%$ of patients on the dasatinib arm and in $74 \%$ of patients on the HD imatinib arm. MMR rates were also more frequent with dasatinib than with HD imatinib (29\% vs $12 \% ; P=0.028)$. The estimated PFS also favored dasatinib $(P=0.0012) .{ }^{26}$ Dasatinib was the first TKI approved to treat patients with imatinib-resistant CML-CP. There were no other randomized clinical trials comparing efficacy of the $2 \mathrm{G}$-TKIs with escalated dose imatinib in standard dose imatinib-resistant patients. However, the rates of cytogenetic and molecular 
responses achieved in patients treated with $2 \mathrm{G}$-TKIs frontline are considerably higher relatively to those achieved by patients treated with HD imatinib (Table 2). The recent ELN guidelines for management of CML-CP do not recommend imatinib dose escalation in case of treatment failure on the standard imatinib dose $e^{4}$ since the 2 G-TKIs (nilotinib, dasatinib, and bosutinib) and the 3G-TKI (ponatinib) appeared to be more effective in such circumstances. ${ }^{27-29}$

\section{High-dose imatinib in AP/BP of CML}

The ELN definitions of AP and BP of CML differ from those recommended by the World Health Organization (WHO), but are used in almost all recent major studies of CML. According to ELN, AP is defined by $15 \%-29 \%$ blast cells or by $30 \%-49 \%$ blast cells plus promyelocytes in blood or bone marrow. BP is defined by a percentage of blast cells $\geq 30 \%$ in blood or bone marrow or by blast cells involvement of nonhematopoietic tissues, excluding liver and spleen. ${ }^{4}$ In the WHO definition, the percentage of blast cells for AP is $15 \%-19 \%$ and $\geq 20 \%$ for BP in blood or bone marrow. Treatment recommendations are different when patients are diagnosed with AP and BP at baseline, prior to any treatment, or when progression occurs during the treatment of CP. HD imatinib is one of the recommended standards in the therapy of advanced-phase CML. Several studies regarding efficacy of imatinib at the dose of $\geq 600 \mathrm{mg}$ daily in AP/BP CML have been published. In AP patients, the reported CHR rates ranged from $40 \%$ to $82 \%$, with MCyR rates between $24 \%$ and $49 \%{ }^{30-32}$ These response rates are low, compared to those achieved in CML-CP, but significantly higher than those provided by IFN- $\alpha$ or any other previous agent. In 2009, Palandri et al presented the outcome of the study on

Table 2 Selected trials comparing standard- or high-dose imatinib or second-generation TKIs in newly diagnosed patients with CML

\begin{tabular}{|c|c|c|c|c|}
\hline Study & $\begin{array}{l}\text { Follow-up } \\
\text { (months) }\end{array}$ & Random & $\begin{array}{l}\text { CCyR } \\
\text { (\%) }\end{array}$ & $\begin{array}{l}\text { MMR } \\
\text { (\%) }\end{array}$ \\
\hline \multirow[t]{2}{*}{ TOPS } & 12 & IM 400 mg & 66 & 40 \\
\hline & & IM 800 mg & 70 & 46 \\
\hline \multirow[t]{2}{*}{ German Study IV } & 12 & IM 400 mg & 49 & 31 \\
\hline & & IM 800 mg & 63 & 55 \\
\hline \multirow[t]{3}{*}{ ENESTnd } & 24 & NILO 300 mg BID & 87 & 62 \\
\hline & & NILO 400 mg BID & 85 & 59 \\
\hline & & IM $400 \mathrm{mg}$ & 77 & 37 \\
\hline \multirow[t]{2}{*}{ DASISION } & 18 & DASA $100 \mathrm{mg}$ & 78 & 46 \\
\hline & & IM 400 mg & 70 & 28 \\
\hline
\end{tabular}

Abbreviations: BID, twice daily; CML, chronic myeloid leukemia; CCyR, complete cytogenetic response; DASA, dasatinib; IM, imatinib; MMR, major molecular response; NILO, nilotinib; TKI, tyrosine kinase inhibitor.
Table 3 Summary of the outcomes of trials with imatinib (from most recent) in CML-BP patients

\begin{tabular}{llll}
\hline $\begin{array}{l}\text { Imatinib } \\
\text { dosage }(\mathbf{m g})\end{array}$ & Study & $\begin{array}{l}\text { Cytogenetic } \\
\text { response (\%) }\end{array}$ & $\begin{array}{l}\text { Overall survival } \\
\text { at I2 months (\%)* }\end{array}$ \\
\hline $400-600$ & ${\text { Silver et al }{ }^{36}}^{36}$ & 9 & NA \\
600 & Palandri et al $^{33}$ & 17 & 29 \\
600 & Sureda et al $^{37}$ & 13 & 36 \\
$400-600$ & Sawyers et al $^{34}$ & 16 & 30 \\
$300-1,000$ & Kantarjian et al $^{31}$ & 16 & 22 \\
$300-600$ & Druker et al $^{38}$ & 12 & NA \\
\hline
\end{tabular}

Note: *Cytogenetic response includes complete, partial, minimal, and minor responses when available.

Abbreviations: CML-BP, blastic phase of chronic myeloid leukemia; NA, not available.

111 patients in newly diagnosed AP treated with imatinib at a dose of $600 \mathrm{mg}$ daily. Imatinib was given in doses of $600 \mathrm{mg}$ daily to all patients. Treatment was continued until disease progression, death, intolerance to imatinib, or allogeneic stem cell transplantation (alloSCT). The median follow-up was 82 months (range: 73-87). In all, 107 patients (96\%) returned to chronic phase and 79 (71\%) achieved CHR. Cumulative best rates of MCyR and CCyR were $30 \%$ and $21 \%$, respectively. All responses were maintained for a minimum of 4 weeks. At last follow-up, four patients were alive in complete remission after allogeneic transplant, 16 patients $(14 \%)$ had switched to a 2 G-TKIs, and 21 patients $(19 \%)$ were alive on imatinib therapy. No late toxicities were observed. PFS and EFS rates were $36.5 \%$ and $15 \%$, respectively, at 7 years. ${ }^{33}$ Sawyers et al conducted the study on 260 patients, of whom 229 had a confirmed diagnosis of CML in myeloid BP. Most of the patients had not received any specific therapy for advanced CML except for IFN and palliative therapy with hydroxyurea or low-dose cytosine arabinoside. When Phase I dose-escalation data demonstrated the safety of prolonged treatment with imatinib at higher doses, the initial daily dose was increased from 400 to $600 \mathrm{mg}$ daily. Imatinib-induced hematologic responses in $52 \%$ of patients and sustained hematologic responses lasting at least 4 weeks in $31 \%$ of patients, including CHR in $8 \%$. For patients with a sustained response, the estimated median response duration was 10 months. Imatinib-induced MCyR in $16 \%$ of patients, with $7 \%$ of the responses being complete. Median survival time was 6.9 months. Nonhematologic adverse reactions were frequent but generally mild or moderate. Episodes of severe cytopenia were also frequent and were attributable to both the underlying condition and the treatment. ${ }^{34}$ Obviously, the development of the $2 \mathrm{G}$ - and recently the $3 \mathrm{G}$ - TKIs has changed the landscape of advanced-phase CML therapy. These agents might be 
effective in patients with $\mathrm{HD}$ imatinib-resistant AP/BP. ${ }^{35}$ The summary of responses to imatinib in BP patients is shown in Table 3. ${ }^{31,33-34,36-38}$ According to the recent ELN recommendations, newly diagnosed TKI-naïve patients with AP or BP should be treated with imatinib $400 \mathrm{mg}$ twice daily, or dasatinib $70 \mathrm{mg}$ twice daily or $140 \mathrm{mg}$ once daily. Subsequently, alloSCT is recommended for all BP patients and for those AP patients who do not achieve an optimal response. AP or BP that develops as a progression from $\mathrm{CP}$ in TKI-pretreated patients should be managed with any of the TKIs that were not used before progression. Then, alloSCT is recommended in all patients, if eligible. ${ }^{4}$

\section{Conclusion - current place in CML therapy}

Modern therapy which radically changed the prognosis of CML started in June 1998 with the first administration of imatinib, a first-generation TKI. The expectations of patients and physicians regarding the results of therapy have been substantially changed over the last decade of experience with TKIs. The treatment goal for CML is no longer a prolongation of survival, but discontinuation of therapy and cure. Deep molecular responses are achieved by the majority of imatinibtreated patients; however, the results of clinical trials suggest that patients treated up-front with $2 \mathrm{G}$-TKIs have a better chance to achieve faster and deeper molecular responses $\left(\mathrm{MR}^{4.0}\right.$ or $\left.\mathrm{MR}^{4,5}\right)$, which are the key criteria for discontinuation studies. Nevertheless, until recently, all the $2 \mathrm{G}-\mathrm{TKI}$ compared to imatinib studies have shown no difference in OS. During the 2014 ASH Annual Meeting, Larson et al presented the latest follow-up of ENEST study in which, after 6 years, there were significantly fewer deaths among patients in the nilotinib $400 \mathrm{mg}$ twice daily arm than in the imatinib $400 \mathrm{mg}$ once daily arm. ${ }^{39}$ Most patients diagnosed with CML will receive prolonged therapy; therefore, individual patient's comorbidities, disease characteristics, ability to follow doctor's orders, and treatment schedule and preferences regarding a goal of therapy should guide the decision of choice of initial treatment. Patients with CML-CP could be stratified with the use of risk stratification scores, such as Sokal, ${ }^{40}$ Hasford, ${ }^{41}$ and EUTOS, ${ }^{42}$ to help predict the outcomes of therapy. Lowrisk category patients are expected to have optimal responses on imatinib, dasatinib, or nilotinib used in the first-line setting. However, patients with intermediate- or high-risk disease may benefit more when $2 \mathrm{G}$-TKIs are chosen as a frontline treatment. ${ }^{43,44}$ Another factor that could influence the choice of optimal initial treatment is human organic cation transporter-1 (OCT-1) expression. Low OCT-1 expression and activity could affect the concentrations of imatinib in CML cells $^{45-47}$ and is associated with lower OS (87\% vs 96\%) and EFS (48\% vs 74\%) when compared to patients with high OCT-1 activity. Therefore, it was suggested that patients with low activity of OCT-1 may benefit from dasatinib or nilotinib since they are not transported into CML cells by OCT-1. The role of innate immunity in the achievement of optimal response to therapy was underlined by observation by Yeung. Killer immunoglobulin-like receptors - specifically, the KIR2DS1 allele has been associated with lower probabilities of achieving CCyR at 2 years, reduced probabilities of OS and PFS, and lower rates of MMR in patients treated with frontline imatinib therapy. ${ }^{48,49}$ Since the results of clinical trials do not support sufficiently the routine use of HD imatinib as frontline treatment for CML-CP all the aforementioned factors could favor a choice of $2 \mathrm{G}-\mathrm{TKI}$ in the first-line setting. A number of analyses have been conducted to determine the response rates of patients resistant to standard dose of imatinib including those with various $B C R-A B L 1$ mutations to HD imatinib and 2G-TKIs. Based on the critical analysis of those studies, the recent ELN recommendation does not support imatinib dose escalation in such cases since the $2 \mathrm{G}$-TKIs and 3G-TKIs appeared to be more effective. ${ }^{24,25} \mathrm{HD}$ imatinib is recommended as one of the possible treatment option for AP and BP of CML. Taking into consideration that clonal evolution with new additional cytogenetic aberrations and newly emerging ABL kinase domain mutations contribute largely to CML progression, the therapy of AP/BP with TKI according to mutational tests, and possibly combination with chemotherapy should be considered.

\section{Acknowledgment}

TS was supported by EU program: FP7-REGPOT-2012CT2012-316254-BASTION.

\section{Disclosure}

The authors report no conflicts of interest in this work.

\section{References}

1. Cherda B, Melo J. Natural course and biology of CML. Ann Hematol. 2015;94(Suppl 2):S107-S121.

2. Druker BJ, Talpaz M, Resta DJ, et al. Efficacy and safety of a specific inhibitor of the BCR-ABL tyrosine kinase in chronic myeloid leukemia. N Engl J Med. 2001;344:1031-1037.

3. Cohen MH, Moses ML, Pazdur R. Gleevec for the treatment of chronic myelogenous leukemia: US Food and Drug Administration regulatory mechanisms, accelerated approval, and orphan drug status. Oncologist. 2002;7(5):390-392.

4. Baccarani M, Deininger MW, Rosti G, et al. European LeukemiaNet recommendations for the management of chronic myeloid leukemia. Blood. 2013;122(6):872-884. 
5. Cortes J, Giles F, O'Brien S, et al. Result of high-dose imatinib mesylate in patients with Philadelphia chromosome-positive chronic myeloid leukemia after failure of interferon-alpha. Blood. 2003;102(1):83-86.

6. Castagnetti F, Palandri F, Amabile M, et al. Results of high dose imatinib mesylate in intermediate Sokal risk chronic myeloid leukemia patients in early chronic phase: a phase 2 trial of the GIMEMA CML Working Party. Blood. 2009;113(15):3428-3434.

7. Kantarjian H, Talpaz M, O’Brien S, et al. High-dose imatinib mesylate therapy in newly diagnosed Philadelphia chromosome-positive chronic phase chronic myeloid leukemia. Blood. 2004;103:2873-2878.

8. Andreas AP, Wolf DW, Fong DF, et al. High doses of imatinib mesylate $(800 \mathrm{mg} /$ day $)$ significantly improve rates of major and complete cytogenetic remissions (MCR, CCR) - results from the first planned interim analysis of a multicenter, randomised, 2 arm phase III study comparing imatinib standard dose (400 mg/day) with imatinib high dose. Haematologica. 2008;93. Abst 0406 (EHA Annual Meeting Abstracts)

9. Hughes TP, Branford S, White DL, et al. Impact of early dose intensity on cytogenetic and molecular responses in chronic-phase CML patients receiving $600 \mathrm{mg}$ /day of imatinib as initial therapy. Blood. 2008;112(10):3965-3973.

10. Hughes TP, Kaeda J, Branford S, et al. Frequency of major molecular responses to imatinib or interferon alpha plus cytarabine in newly diagnosed chronic myeloid leukemia. N Engl J Med. 2003;349: 1423-1432.

11. Druker BJ, Guilhot F, O’Brien SG, et al. Five-year follow-up of patients receiving imatinib for chronic myeloid leukemia. $N$ Engl $\mathrm{J} \mathrm{Med}$. 2006;355:2408-2417.

12. Picard S, Titier K, Etienne G, et al. Trough imatinib plasma levels are associated with both cytogenetic and molecular responses to standard dose imatinib in chronic myeloid leukemia. Blood. 2007; 109(8):3496-3499.

13. Yeung DT, Osborn MP, White DL, et al. TIDEL-II: first-line use of imatinib in CML with early switch to nilotinib for failure to achieve time-dependent molecular targets. Blood. 2015;125(6):915-923.

14. Gafter-Gvili A, Leader A, Gurion R, et al. High-dose imatinib for newly diagnosed chronic phase chronic myeloid leukemia patients-systematic review and meta-analysis.AmJHematol.2011;86(8) 657-662.

15. Baccarani M, Rosti G, Castagnetti F, et al. Comparison of imatinib $400 \mathrm{mg}$ and $800 \mathrm{mg}$ daily in the front-line treatment of high-risk, Philadelphia-positive chronic myeloid leukemia: a European Leukemia Net Study. Blood. 2009;113:4497-4504.

16. Cortes JG, Baccarani M, Guilhot F, et al. First report of the TOPS study: a randomized phase III trial of $400 \mathrm{mg}$ vs $800 \mathrm{mg}$ imatinib in patients with newly diagnosed, previously untreated CML in chronic phase using molecular endpoints. Haematologica. 2008;93(Suppl 1):160. Abstract 0402.

17. Preudhomme C, Guilhot J, Nicolini FE, et al. Imatinib plus peginterferon alpha-2a in chronic myeloid leukemia. N Engl J Med. 2010;363: 2511-2521.

18. Hehlmann R, Lauseker M, Jung-Munkwitz S, et al. Tolerability-adapted imatinib $800 \mathrm{mg} / \mathrm{d}$ versus $400 \mathrm{mg} / \mathrm{d}$ versus $400 \mathrm{mg} / \mathrm{d}$ plus interferonalpha in newly diagnosed chronic myeloid leukemia. J Clin Oncol. 2011;29(12):1634-1642.

19. Marin D, Goldman JM, Olavarria E, Apperley JF. Transient benefit only from increasing the imatinib dose in CML patients who do not achieve complete cytogenetic remissions on conventional doses. Blood. 2003;102:2702-2703.

20. Zonder JA, Pemberton P, Brandt H, et al. The effect of dose increase of imatinib mesylate in patients with chronic or accelerated phase chronic myelogenous leukemia with inadequate hematologic or cytogenetic response to initial treatment. Clin Cancer Res. 2003;9: 2092-2097.

21. Baccarani M, Cortes J, Pane F, et al. Chronic myeloid leukemia: an update of concepts and management recommendations of European Leukemia Net. J Clin Oncol. 2009;27:6041-6051.
22. Kantarjian HM, Larson RA, Guilhot F, et al. International randomized study of interferon vs STI571 (IRIS) investigators efficacy of imatinib dose escalation in patients with chronic myeloid leukemia in chronic phase. Cancer. 2009;115:551-560.

23. Breccia M, Stagno F, Vigneri P, et al. Imatinib dose escalation in 74 failure or suboptimal response chronic myeloid leukaemia patients at 3-year follow-up. Am J Hematol. 2010;85(5):375-377.

24. Jabbour E, Kantarjian H, Jones D, et al. Imatinib mesylate dose escalation is associated with durable responses in patients with chronic myeloid leukemia after cytogenetic failure on standard-dose imatinib therapy. Blood. 2009;113:2154-2160.

25. Kantarjian H, Pasquini R, Hamerschlak N, et al. Dasatinib or high-dose imatinib for chronic-phase chronic myeloid leukemia after failure of first-line imatinib: a randomized phase 2 trial. Blood. 2007;109(12): 5143-5150.

26. Kantarjian H, Pasquini R, Levy V, et al. Dasatinib or high-dose imatinib for chronic-phase chronic myeloid leukemia resistant to imatinib at a dose of 400 to 600 milligrams daily: two-year follow-up of a randomized phase 2 study (START-R). Cancer. 2009;115(18):4136-4147.

27. Kantarjian H. Dasatinib versus imatinib in newly diagnosed chronicphase chronic myeloid leukemia. $N$ Engl J Med. 2010;362(24): 2260-2270.

28. Saglio G, Kim DW, Issaragrisil S, et al. Nilotinib versus imatinib for newly diagnosed chronic myeloid leukemia. $N$ Engl J Med. 2010; 362(24):2251-2259.

29. Hochhaus A, Ernst T, Eigendorf E, Rosee PE. Causes of resistance and treatment choices of second- and third-line treatment in chronic myelogenous leukemia patients. Ann Hematol. 2015;94(Suppl 2): S133-S140.

30. Silver RT, Talpaz M, Sawyers CL, et al. Four years of follow-up of 1027 patients with late chronic phase (LCP), accelerated phase (AP), or blast crisis (BC) chronic myeloid leukemia (CML) treated with imatinib in three large phase II trials. Blood. 2004;104:23.

31. Kantarjian HM, O'Brien S, Cortes JE, et al. Treatment of Philadelphia chromosome-positive, accelerated-phase chronic myelogenous leukemia with imatinib mesylate. Clin Cancer Res. 2002;8:2167-2176.

32. Talpaz M, Silver RT, Druker BJ, et al. Imatinib induces durable hematologic and cytogenetic responses in patients with accelerated phase chronic myeloid leukemia: results of a phase 2 study. Blood. 2002;99: 1928-1937.

33. Palandri F, Castagnetti F, Alimena G, et al. The long-term durability of cytogenetic responses in patients with accelerated phase chronic myeloid leukemia treated with imatinib $600 \mathrm{mg}$ : the GIMEMA CML Working Party experience after a 7-year follow-up. Hematologica. 2009;94(2):205-212.

34. Sawyers CL, Hochhaus A, Feldman E, et al. Imatinib induces hematologic and cytogenetic responses in patients with chronic myelogenous leukemia in myeloid blast crisis: results of a phase II study. Blood. 2002;99(10):3530-3539.

35. Cortes J, Kim DW, Raffoux E, et al. Efficacy and safety of dasatinib in imatinib-resistant or -intolerant patients with chronic myeloid leukemia in blast phase. Leukemia. 2008;22(12):2176-2183.

36. Silver RT, Cortes J, Waltzman R, et al. Sustained durability of responses and improved progression-free and overall survival with imatinib treatment for accelerated phase and blast crisis chronic myeloid leukemia: long-term follow-up of the STI571 0102 and 0109 trials. Haematologica. 2009;94:743-744.

37. Sureda A, Carrasco M, deMiguel M, et al. Imatinib mesylate as treatment for blastic transformation of Philadelphia chromosome positive chronic myelogenous leukemia. Haematologica. 2003;88:1213-1220.

38. Druker B. Signal transduction inhibition: results from phase I clinical trials in chronic myeloid leukemia. Semin Hematol. 2001;38:9-14.

39. Larson RA, Kim D-W, Issaragrilsil S, et al. Efficacy and safety of nilotinib (NIL) vs imatinib (IM) in patients (pts) with newly diagnosed chronic myeloid leukemia in chronic phase (CML-CP): long-term follow-up (f/u) of ENESTnd. Blood. 2014;124(21). Abst 4541 (ASH Annual Meeting Abstracts). 
40. Sokal JE, Cox BE, Baccarani M, et al. Prognostic discrimination in “Good-Risk" chronic granulocytic leukemia. Blood. 1984;63(4):789.

41. Hasford J, Pfirmann M, Hehlmann R, et al. A new prognostic score for survival of patients with chronic myeloid leukemia treated with interferon alpha. J Natl Cancer Inst. 1998;90:850-858.

42. Hasford J, Baccarani M, Hoffmann V, et al. Predicting complete cytogenetic response and subsequent progression-free survival in 2060 patients with CML on imatinib treatment: the EUTOS score. Blood. 2011;118(3):686-692.

43. Jabbour E, Kantarjian HM, Saglio P, et al. Early response with dasatinib or imatinib in chronic myeloid leukemia: 3-year follow-up from a randomized phase 3 trial (DASISION. Blood. 2014;123(4):494-500.

44. Kantarjian HM, Hochhaus A, Saglio G, et al. Nilotinib versus imatinib for the treatment of patients with newly diagnosed chronic phase, Philadelphia chromosome-positive, chronic myeloid leukaemia: 24-month minimum follow-up of the phase 3 randomised ENESTnd trial. Lancet Oncol. 2011;12(9):841-851.

45. White DL, Saunders VA, Dang P, et al. Most CML patients who have a suboptimal response to imatinib have low OCT-1 activity: higher doses of imatinib may overcome the negative impact of low OCT-1 activity. Blood. 2007; 110:4064-4072.
46. White DL, Dang P, Engler J, et al. Functional activity of the OCT-1 protein is predictive of long-term outcome in patients with chronicphase chronic myeloid leukemia treated with imatinib. J Clin Oncol. 2010;28:2761-2739.

47. White DL, Radich J, Soverini S, et al. Chronic phase chronic myeloid leukemia patients with low OCT-1 activity randomized to high-dose imatinib achieve better responses, and lower failure rates, than those randomized to standard-dose. Haematologica. 2012;97:907-914.

48. Yeung DT, Vidovic L, Tang C, et al. KIR2DL5B genotype independently predicts poor outcomes in CML-CP patients switched to nilotinib after suboptimal responses to imatinib and may refine prognosis in patients with EMR failure. Blood. 2014;124(21):814.

49. Marin D, Gabriel IH, Ahmad S, et al. KIR2DS1 genotype predicts for complete cytogenetic response and survival in newly diagnosed chronic myeloid leukemia patients treated with imatinib. Leukemia. 2012;26:296-302.

\section{Dovepress}

\section{Publish your work in this journal}

Blood and Lymphatic Cancer: Targets and Therapy is an international, peer-reviewed, open access journal focusing on blood and lymphatic cancer research, identification of therapeutic targets and the optimal use of preventative and integrated treatment interventions to achieve improved outcomes, enhanced survival and quality of life for the cancer patient. The manuscript management system is completely online and includes a very quick and fair peer-review system. Visit http://www.dovepress.com/testimonials.php to read real quotes from published authors. 